\title{
Incidence and Risk Factors of Acute Kidney Injury in ICU Patients of Omdurman Teaching Hospital
}

\author{
Abdel Mumin Sid Ahmed, Nusaiba Hassan Mohamed Eltahir \\ Omdurman teaching Hospital, Omdurman, Khartoum State, Sudan \\ Email: mumin.sidahmed@outlook.com, Nusaibahassan1212@gmail.com
}

How to cite this paper: Ahmed, A.M.S. and Eltahir, N.H.M. (2021) Incidence and Risk Factors of Acute Kidney Injury in ICU Patients of Omdurman Teaching Hospital. Open Journal of Nephrology, 11, 43-57. https://doi.org/10.4236/ojneph.2021.111005

Received: January 28, 2021

Accepted: March 12, 2021

Published: March 15, 2021

Copyright $\odot 2021$ by author(s) and Scientific Research Publishing Inc. This work is licensed under the Creative Commons Attribution International License (CC BY 4.0).

http://creativecommons.org/licenses/by/4.0/

\begin{abstract}
Background: Acute kidney injury (AKI) is a common complication in critically ill patients and it is commonly associated with high mortality and morbidity with adverse short and long term outcome. Objectives: The main objective of this study was to determine the incidence of acute kidney injury in ICU patients in Omdurman teaching hospital, and to assess the risk factors associated with the development of acute kidney injury in a critically ill patient. We also intended to find out the impact of acute kidney injury on ICU outcomes. Methods: In this descriptive prospective, cohort hospital-based study, 211 patients were studied and followed up during admission in ICU from November 2019 until July 2020 in Omdurman teaching hospital. Results: 61 patients of the total 211 patients developed renal impairment during their stay in ICU. 64\% (39 patients) were males and 36\% (22 patients) were female, mean age was $41 \pm 5$, renal impairment developed after a mean of 5 days of admission and the most common cause was decreased level of consciousness and severe hypotension, sepsis accounting for $40 \%$ of the admission. The outcome in ICU showed that the mortality rate was high up to $41 \%$ in patients who developed acute kidney injury, Sepsis accounted for over $96 \%$ (24 patients) who have passed away. Conclusions: Development of AKI in ICU is an important risk factor for poor outcomes in critically setting. Prevention is the best method for avoiding AKI, with the early identification and recognition of high-risk patients.
\end{abstract}

\section{Keywords}

Acute Kidney Injury, ICU, Risk Factors, Outcome, Frequency

\section{Introduction}

AKI is a common complication in patients admitted to the intensive care unit 
(ICU) and is commonly associated with adverse outcomes, increasing the length of ICU stay, increasing short and long term mortality, and contributing to developing chronic kidney disease (CKD).

The identification and studying of the risk factors associated with the development of acute kidney injury will help in providing and establishing preventive measure that could improve survival in those patients. As recognition of those high-risk patients will allow developing steps and guidelines help in prevention, diagnosis and treatment of AKI.

Although acute kidney injury is preventable and treatable in most cases, it carries a high mortality rate in a critical care setting, especially in developing countries. This research was focused on the frequency, risk factors, and outcome.

\section{Literature Review, Rationale \& Objectives}

\subsection{Literature Review}

Over the past decades, there was an increased incidence of acute kidney injury, and given the adverse outcomes especially in the long term, AKI is now a growing concern for health care worldwide.

Acute kidney injury is a challenging medical complication with hospital costs exceeded only by those for acute myocardial infarction and stroke. This condition is a sudden decline in kidney function during hours to days originating in either the community or hospital setting [1]. Mortality rates progress proportionally with the severity of the decrease in kidney function [2]. Acute renal injury defined as 1) Increase in serum creatinine by $0.3 \mathrm{mg} / \mathrm{dL}$ or more within 48 hours or 2) Increase in serum creatinine to 1.5 times baseline or more within the last 7 days or 3) Urine output less than $0.5 \mathrm{~mL} / \mathrm{kg} / \mathrm{h}$ for 6 hours using Kidney Disease Improving Global Outcomes (KDIGO) [3].

In the hospital setting, patients with AKI have lengths of stay 2.4 times longer than patients who do not have AKI Hospital mortality rates are as high as $60 \%$ in ICU patients and $80 \%$ in patients requiring renal replacement therapy (RRT).

\subsection{Risk Factors}

The potential risk factors include sex, race, reference creatinine, estimated glomerular filtration rate (eGFR) derived from the reference creatinine.

Elderly patients are at risk of AKI due to decreased renal reserve and altered kidney function inhibiting kidney function recovery following acute injury. The incidence of AKI has increased steadily in recent years, and this increase is associated strongly with advancing age in the population because epidemiologic data indicate that $\mathrm{AKI}$ is much more common in the elderly. In the ageing population, there is heightened susceptibility to drug toxicity, partially owing to altered drug pharmacokinetics and pharmacodynamics. Furthermore, the elderly consume twice as many medications overall, including nephrotoxic agents, compared with younger patients [3].

Severe sepsis is one of the leading causes of acute kidney injury (AKI). Pa- 
tients with sepsis-associated AKI demonstrate high-hospital mortality. During the past decades, the incidence of severe sepsis has increased. A study reported a 75\% increase in ICU treated severe sepsis from 1993 to 2001 in France [4]. Of patients with sepsis in the ICU, $31 \%$ - $61 \%$ have AKI [5].

Overall, the prevalence of AKI in the setting of MI is $16 \%$. Patients with AKI had a substantially higher risk of mortality than those without AKI. Patients with AKI are at a substantially higher risk of in-hospital major bleeding events, despite lower rates of invasive strategies [6].

Studies have recently shown that up to $40 \%$ of the population has CKD, and this confers up to the 8 -fold increased risk of mortality among affected patients [7]

Diabetes is the most frequent cause of end-stage renal disease in our society. Acute kidney injury remains a clinical and prognostic problem of fundamental importance since incidences have been increased in recent years, while mortality has not substantially been improved [8]. Approximately $40 \%$ of all patients requiring dialysis therapy on a regular basis suffer from diabetes mellitus as the respective cause [9].

Diabetic kidney disease, commonly termed diabetic nephropathy (DN) is the most common cause of the end-stage renal disease (ESRD) worldwide. The characteristic histopathology of DN includes glomerular basement membrane (GBM) thickening, mesangial expansion, nodular glomerular sclerosis, and tubulointerstitial fibrosis. Diabetes is associated with many metabolic derangements, such as reactive oxygen species (ROS) overproduction, hypoxic state, mitochondrial dysfunction, and inflammation [10].

Multiple studies have shown that diabetes alone is an independent risk for acute kidney injury [11] [12]. The incidence of AKI was found to be higher in diabetic patients undergoing surgery [13]. Renal insults resulting in tissue injury, including acute tubular injury, may affect kidney function and the rate of chronic functional impairment in diabetic patients since the subsequent maladaptive recovery fails to fully reverse the insults.

Aminoglycosides are potent bactericidal antibiotics that are highly effective against gram-negative bacterial infections. They have increasingly been used as gram-negative organisms have become progressively more resistant to beta-lactam antibiotics and fluoroquinolones.

They were recently found to be prescribed in $12.1 \%$ of the cases in which an antibiotic was necessary for the ICU [14].

The most important adverse effects of aminoglycosides are nephrotoxicity, ototoxicity, and, more rarely, neuromuscular blockade. Renal injury can occur in a substantial number of patients receiving an aminoglycoside, and whether it occurs depends on the patient's clinical condition and interactions with other nephrotoxic drugs [15] [16] Aminoglycoside-associated kidney injury may manifest as a decrease in the glomerular filtration rate, enzymatic, aminoaciduria, glycosuria, hypomagnesemia, hypocalcemia, and hypokalemia. Fanconi-like and 
Bartter-like syndromes, as well as impaired renal concentrating mechanisms, have also been described [15].

Several risk factors for aminoglycoside-associated nephrotoxicity have been identified, including the presence of comorbidities, volume depletion, liver dysfunction, sepsis, renal dysfunction, hypokalemia, hypomagnesemia, advanced age, prolonged therapy, the type of aminoglycoside, the frequency of aminoglycoside dosing, an elevated serum aminoglycoside concentration, the timing of aminoglycoside administration, the simultaneous consumption of other medications, and interactions with other nephrotoxic drugs [17].

\subsection{Rationale}

According to our knowledge, and local data available we found there were no study has been conducted in our region that investigating the incidence and risk factors of developing renal injury as the problem among ICU patients so, for this reason, we intended to carry out this study as it will add a huge knowledge and advances in management and control of this disease.

\section{Objectives}

\subsection{Main Objective}

The main objective of this study was to determine the incidence of acute kidney injury in ICU patients in Omdurman teaching hospital.

\subsection{Specific Objectives}

1) To determine the incidence of acute kidney injury in ICU patients.

2) To assess the risk factors associated with the development of acute kidney injury in critically ill patients.

4) To find out the impact of acute kidney injury on ICU outcomes.

\subsection{The Research Problem}

Some patients admitted to ICU with no history of kidney disease developed acute kidney injury and required urgent intervention which significantly affects the outcome and course of treatments for those patients.

\section{Methods}

\subsection{Study Area}

The study was conducted from November 2019 until July 2020 in Omdurman teaching hospital, ICU department.

Omdurman teaching hospital is a tertiary hospital work at the level of major medical and surgical specialities. The ICU department consists of major room accommodate Eight ICU beds for general medicine and surgical patients and small room specialized for septic patients. The staff of ICU consists of three intensivists, two medicine specialist and 21 ICU residents. 


\subsection{Study Design}

It was a prospective, cohort hospital-based study.

\subsection{Study Population}

Adult Patients admitted to the ICU department for more than 24 hours with normal renal profile.

\subsection{Exclusion Criteria}

Patient with the previous history of renal impairment or admitted with a condition known to cause kidney injuries such as poising or drugs overdose.

\subsection{Data Collection Techniques}

Secondary Data was collected using a checklist and datasheet to collect data from files and medical records. The data collected by trained volunteer following local ICU protocols of infection controls, this data sheet was containing demographic data and history of the illness. Demographic data as age sex resident occupation chronic disease, ICU cause of admission, ICU Score simplified acute physiology score and lab investigation result has been recorded for every patient since day 1 of ICU admission, all the patients assessed in a regular interval after 48. The risk factors included in the analysis were significant predictors of AKI in previous studies [18] and [19].

\subsection{Sampling Technique}

The sample was total coverage of all the patients' eligible for the study.

\subsection{Ethical Consideration}

The proposal of the study has been presented to the ethical Clearance committee of the Khartoum ministry of health and approved. Hospital directors and participants informed about the purposes and objectives of the study and voluntary consent obtained. The privacy of the patient has been strictly maintained all throw the study no information shared with others or used in different research.

\subsection{Data Analysis}

Statistical analyses were performed with SPSS Statistics (version 25.0, SPSS Inc., Chicago, IL, USA). Continuous variables were given as means and standard deviation provided. Categorical variables were summarized by percentages. Chi-square tests have been used for categorical variables, $t$-tests for normally distributed variables and Mann-Whitney tests for non-parametric variables for between-group comparisons. A P $<0.05$ was considered significant.

\section{Result}

\subsection{General Patient Characteristics}

Table 1 shows the demographic and clinical characteristics of the ICU patient's 
Table 1. Demographic and clinical characteristics of ICU patient's population.

\begin{tabular}{|c|c|}
\hline Variables & All cases, N 211 \\
\hline Gender $(M / F), n$ & $123 / 88$ \\
\hline Age, years, Mean (SD) & $45(10)$ \\
\hline Height cm, Mean (SD) & $171(5)$ \\
\hline Weight kg, Mean (SD) & $76(5)$ \\
\hline \multicolumn{2}{|l|}{ Comorbidities } \\
\hline Diabetes, $\mathrm{n}(\%)$ & $47(22 \%)$ \\
\hline Hypertension, $\mathrm{n}(\%)$ & $32(14 \%)$ \\
\hline Respiratory disease, $\mathrm{n}(\%)$ & $15(7 \%)$ \\
\hline GIT disease, $\mathrm{n}(\%)$ & $7(3 \%)$ \\
\hline Cardiac disease & $2(22 \%)$ \\
\hline CNS disease & $2(22 \%)$ \\
\hline \multicolumn{2}{|l|}{ Total days spent in ICU } \\
\hline Mean, $\mathrm{n}(\mathrm{SD})$ & $9(5)$ \\
\hline Minimum & 2 \\
\hline maximum & 45 \\
\hline \multicolumn{2}{|l|}{ Causes of Admission to ICU, $\mathrm{n}(\%)$} \\
\hline Brain Hemorrhage & $35(17 \%)$ \\
\hline DKA & $29(29 \%)$ \\
\hline Post-Operative & $19(9 \%)$ \\
\hline Septic Shock & $18(8 \%)$ \\
\hline GBS & $17(8 \%)$ \\
\hline Stroke & $16(8 \%)$ \\
\hline Meningoencephalitis & $13(6.2 \%)$ \\
\hline Pulmonary Embolism & $11(5 \%)$ \\
\hline Cerebral Malaria & $9(4 \%)$ \\
\hline Road Traffic & $8(4 \%)$ \\
\hline \multicolumn{2}{|l|}{ The outcome in ICU admission } \\
\hline Improvement & $146(69 \%)$ \\
\hline passed away & $65(31 \%)$ \\
\hline
\end{tabular}

$\mathrm{M} / \mathrm{F}=$ male/female $; \mathrm{SD}=$ Stranded Deviation, GIT $=$ gastrointestinal tract, $\mathrm{CNS}=$ central nervous system, DKA = diabetic ketoacidosis. GBS Gillian bare syndrome. Values are n (\%) unless otherwise specified.

population. During the study period, 211 patients were included. 58\% male (123 Patients), and $42 \%$ female ( 88 patients) minimum age was 18 and the maximum was 95 with a mean age of $45 \pm 10$ years. Weight and height were documented with mean $76 \pm 5$ and $171 \pm 5$ respectively.

Comorbidities also we included in this study. 22\% (47 patients) were diabetics, 14\% (32 patients) were hypertensive, 7\% (15 patients) reported a respiratory condition mostly asthma and COPD, $4 \%$ (7 patients) had a gastrointestinal condition mostly heartburn, irritable bowel syndrome and one patient had peptic ulcer, only 2 patients had epilepsy and 2 patient had previous ischemic heart disease.

The mean of Total days spent in ICU was $9 \pm 2$ days in this study minimal stays as short as 2 days and maximum stay as long as 45 days. the most common Causes of Admission to ICU was decreased level of consciousness and reported 
in $33.6 \%$ (71 patients) followed by observation in $29 \%$ (61) and severe acidosis in $15 \%$ (31 patients) and severe hypoxia in 12\% (26 patients) and severe hypotension in $6 \%$ (12 patients) and lastly post-cardiac arrest in $5 \%$ (10 patients).

The outcome in ICU admission showed that $69 \%$ (146 patients) showed signs of improvement after admission and discharged from ICU while 31\% (65 patients) passed away.

The most common diagnosis is brain hemorrhage irrespective of the cause is 17\% (35 patients) then Diabetic Keto Acidosis (DKA) in 14\% (29 patients) then post-operative observation in 9\% (19 patients) septic shock in $8 \%$ (18 patients) and Guillain-Barre syndrome (GBS) 8\% (17 patients) stroke 8\% (16 patients) Meningoencephalitis 6.2\% (13 patients) and pulmonary embolism 5\% (11 patients) cerebral malaria $4 \%$ ( 9 patients) road traffic in $4 \%$ (8 patients).

The overall ICU mortality was $31 \%$, for patients with renal impairment it was $41 \%$ which was greater than the $23 \%$ mortality of those patients with normal renal function.

\subsection{Renal Impairments}

61 patients (29\%) involved in the study developed renal impairment during their stay in ICU. 39 (64\%) patients were males and 22 (36\%) were female. mean age was $41 \pm 5$ with maximum age was 79 and the minimum was 21 , mean weight and height was $76 \mathrm{~kg}$ and $175 \mathrm{~cm}$ respectively.

Total days spent in ICU was ranging from a minimum as 3 days and maximum as long as 20 days with a mean of 10 days while renal impairment developed after mean of 5 days after admission with a maximum of 9 days and a minimum of 3 days (Table 2).

Table 2. Demographic and clinical characteristics of ICU patient's population who developed acute kidney injury.

\begin{tabular}{lc}
\hline \multicolumn{1}{c}{ Variables } & All cases, N 61 \\
\hline Gender (M/F), $\mathrm{n}$ & $39 / 22$ \\
Age, years, Mean (SD) & $41(5)$ \\
Height cm, Mean (SD) & $175(5)$ \\
Weight kg, Mean (SD) & $76(5)$ \\
Comorbidities & \\
Diabetes, $\mathrm{n}(\%)$ & $19(31 \%)$ \\
Hypertension, $\mathrm{n}(\%)$ & $9(14 \%)$ \\
Respiratory disease, $\mathrm{n}(\%)$ & $4(7 \%)$ \\
GIT disease, $\mathrm{n}(\%)$ & $3(5 \%)$ \\
Cardiac disease & $3(5 \%)$ \\
CNS disease & $1(2 \%)$ \\
& \\
Total days spent in ICU & \\
Mean, $\mathrm{n}(\mathrm{SD})$ & $10(5)$ \\
Minimum & 3 \\
maximum & 20 \\
\hline
\end{tabular}




\section{Continued}

$\begin{array}{lc}\text { Causes of Admission to ICU, } \mathrm{n}(\%) & \\ \text { brain haemorrhage } & 9(15 \%) \\ \text { DKA } & 15(25 \%) \\ \text { post-operative } & 7(11 \%) \\ \text { septic shock } & 25(40 \%) \\ \text { road traffic } & 5(9 \%)\end{array}$

The outcome in ICU admission

$\begin{array}{lc}\text { Improvement } & 29(48 \%) \\ \text { passed away } & 25(41 \%) \\ \text { discharged with deficit } & 7(11 \%)\end{array}$

Cause of admission to ICU (Figure 1) showed the most common cause was decreased level of consciousness $36 \%$ (22 patients), severe hypotension $20 \%$ (12 patients) followed by severe acidosis $18 \%$ (11 patients), 16\% (10 patients) admitted for observation of vitals and $7 \%$ (4 patients) with severe hypoxia and only $3 \%$ (2 patients) admitted after had cardiac arrest irrespective of the cause.

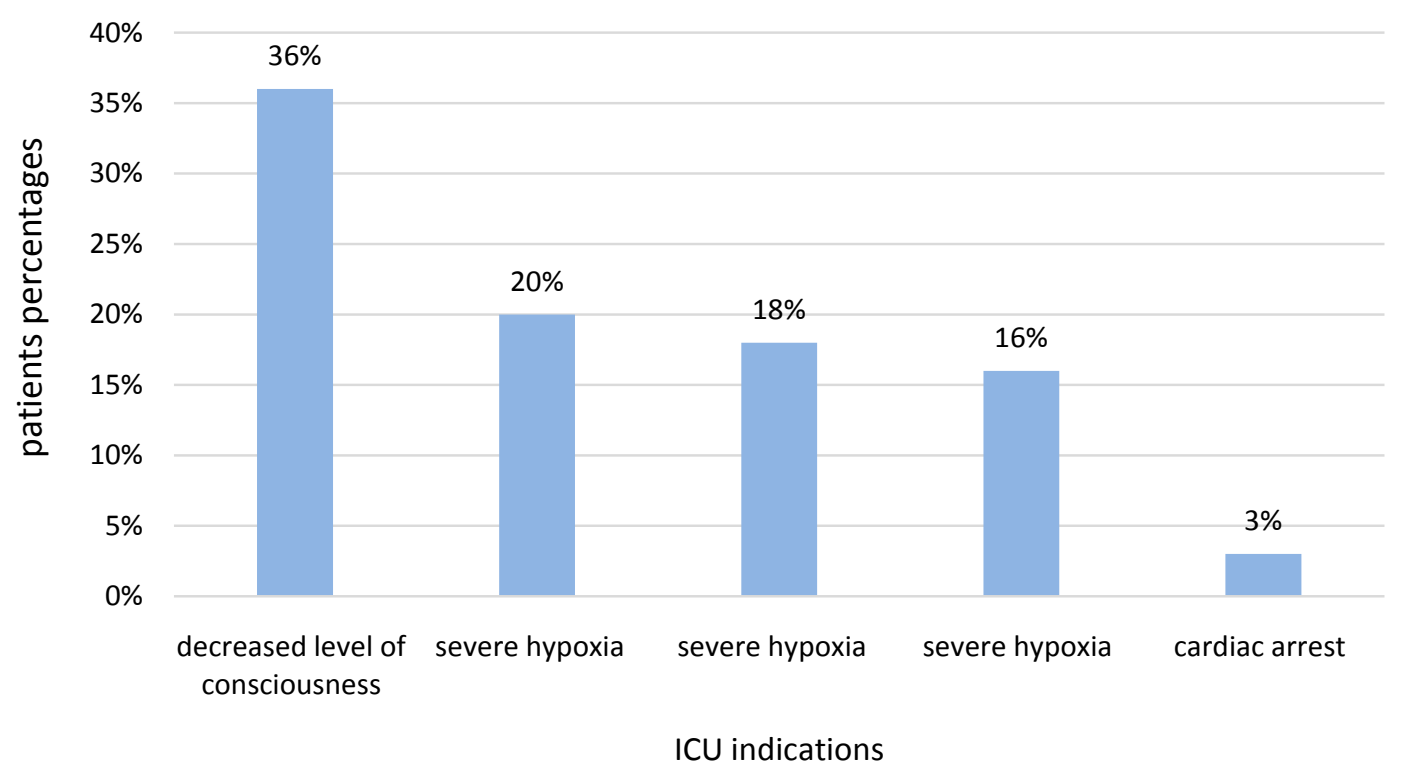

Figure 1. Showed percentage of the most common indication for admission to ICU in patients who developed AKI.

The most common diagnosis admitting the patient to ICU was sepsis accounting for $40 \%$ (25 patients), followed by diabetic ketoacidosis $25 \%$ (15 patients), post-operative recovery $15 \%$ ( 9 patients), and brain haemorrhage accounting for $11 \%$ (7 patients) and least road traffic accident was $9 \%$ (5 patients) (Figure 2).

45\% (28 patients) developed renal impairment while receiving inotropes in ICU. 44\% (27 patients) received a mechanical ventilator Statistical Analysis showed significant differences between the patients developed renal impairment and who revived inotropes and mechanical ventilators $(0.005)$ and (0.007) re- 
spectively.

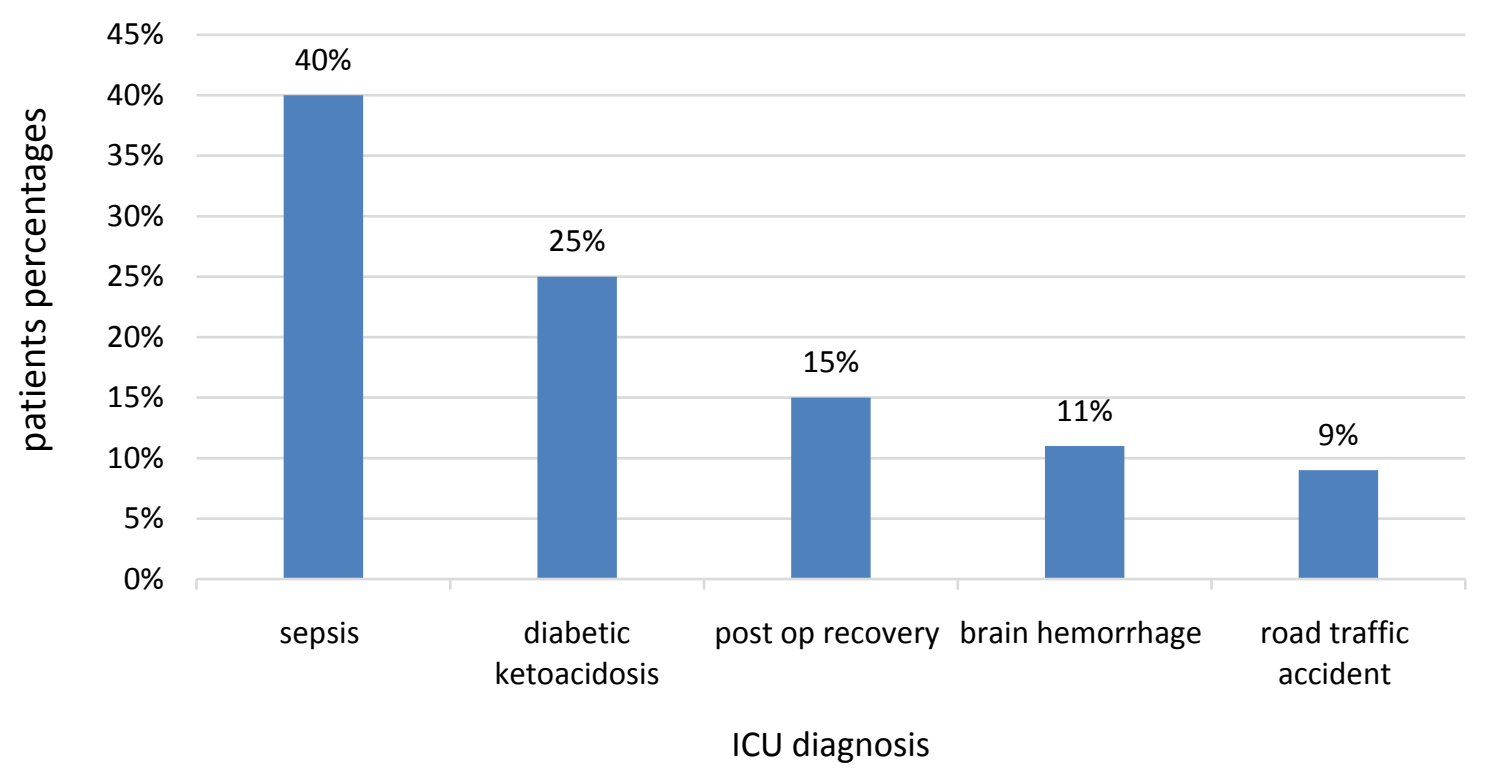

Figure 2. Showed percentage of the most common diagnosis in patients who developed AKI in ICU.

Sepsis and hypovolemia were the most frequent reported etiologies for AKI. Accounting for 51 patients other causes include nephrotoxic drugs (including gentamycin, vancomycin, and contrast dye) causes 6 patients and 4 patients due to rhabdomyolysis after a road traffic accident.

\section{Renal replacement therapy}

Of the 61 causes of AKI, 38 patients had received renal replacement therapy (RRT) either hemodialysis or peritoneal dialysis with a $45 \%$ Mortality, while 23 patients were managing conservatively with a $36 \%$ Mortality (Table 3 ). The majority of RRT procedures were emergency dialysis through a temporary catheter for 2 hours duration.

Table 3. Shows percentages of patients received renal replacement therapy and who managed conservatively in relationship with mortality percentages.

\begin{tabular}{ccc}
\hline & N (\%) & MORTALITY \\
\hline Renal Replacement Therapy & $38(62 \%)$ & $45 \%$ \\
Conservative treatment & $23(38 \%)$ & $36 \%$ \\
\hline
\end{tabular}

\section{Outcome}

The outcome in ICU showed 48\% (29 patients) recovered from renal impairment, $41 \%$ (25 patients) has the past way and 11\% (7 patients) discharged with long term renal replacement therapy afterwards (Figure 3). Statistical Analysis showed significant differences between the outcome and total days spent in ICU (0.003). Sepsis accounts for over 96\% (24 patients) who have passed away ( $\mathrm{p}$ $0.004)$. 


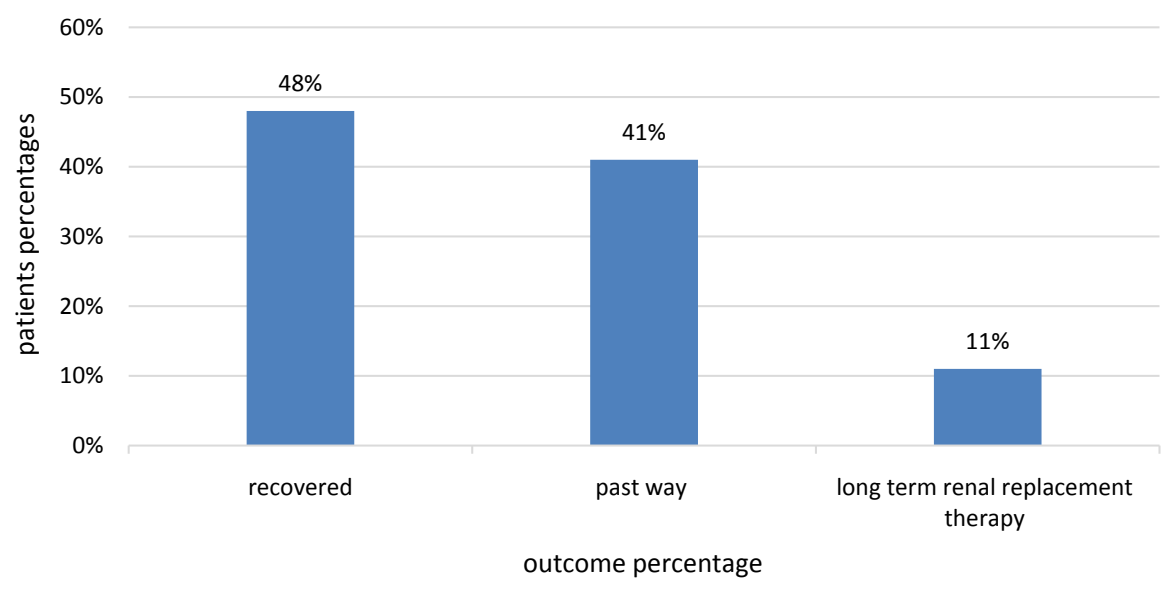

Figure 3. Showed patients percetages of the outcome in patients who developed AKI in ICU.

\section{Discussion}

The aim of this study was not only to determine how frequent renal impairments, is But also to assess and identify the risk factors associated with the development of AKI in those critically ill patients and to find out the impact of developing acute kidney injury on the general ICU outcomes. Early recognition of those high-risk patients provides an opportunity to develop strategies for prevention, diagnosis and early therapeutic intervention.

In this study, we observed 211 selected patient for the study and found that almost $29 \%$ of those patients had developed acute kidney injury, with a mean age of $41 \pm 5$ with a mortality up to $41 \%$.

Literature reviews [20] reported in a large multi-centre study that the incidence of AKI was $39.3 \%$ and mortality was $33.7 \%$ which is lower than our estimation probably due to advanced caregiver to the patients compared to our care the patients received in our ICU. Another study [21], Estimates of AKI prevalence range from $<1 \%$ to $66 \%$ and explained These variations be population differences and the use of AKI classification criteria.

In our study, some factors were observed associated with a greater likelihood of developing acute kidney injury. One of these factors is the length of stay in ICU, the result supports our theory that the longer patient stays increase like-hood of developing acute kidney injury. Our result was significant ( $\mathrm{p}=$ 0.003). We thought this is might be due to increasing the risk espouse to the patient in ICU that the longer patient stays in ICU the longer chance to be exposed to infection and sepsis, nephrotoxic drugs, or stress. this is also observed in a study [22] as reported in Patients who developed AKI had higher APACHE II score and longer ICU stays $(\mathrm{P}<0.001)$, and higher mortality rates $(\mathrm{P}<0.001)$, our result also support the Chinese study [23] A retrospective study across China found AKI was associated with longer length of stay and higher daily costs [23].

The use of invasive mechanical ventilator and inotropes were associated with 
increased incidence and mortality in this study. This association might also reflect the severity of illness in a subset of our patients having other organ system failure leading to mortality. $44 \%$ of patients with AKI have been used an invasive mechanical ventilator and the result showed a significant impact on the outcome $(\mathrm{p}=0.007)$ this was also observed in the study [3] which reported that Invasive mechanical ventilation is associated with a threefold increase in odds of acute kidney injury in critically ill patients also AKI appears to develop more often in patients with invasive Mechanical Ventilators than in those with Non-Invasive Ventilations (NIV). Another study [24] correlate the same finding and reported $21 \%$ of the patients subsequently developed AKI, but without significant difference between ventilation strategies [25].

Our study reported a $45 \%$ of the patients developed AKI have received inotropes which was a significant result $(\mathrm{p}=0.005)$, and Norepinephrine is the most commonly used vasopressor in our patients along with dopamine and epinephrine. This observation can be explained by the fact that the pathophysiology of hypotension and its effect on kidney perfusion will significantly produce a reduction in renal perfusion and overwhelm autoregulation and precipitate an acute fall in Glomerular filtration rate (GFR). These mechanisms have been described in [26].

In our study, we found sepsis is the most common diagnosis required the patient to be admitted to ICU. In around $40 \%$ of the patients, we observed that sepsis is the leading cause and responsible for almost $84 \%$ of the mortality causes of sepsis observed in the present study due to pneumonia, urosepsis and Meningoencephalitis. Many studies have demonstrated the association between sepsis and mortality. In a study [27] Incidence reported Sepsis was considered the cause of acute kidney injury in (47.5\%) of the patients and it was associated with greater aberrations in hemodynamics and laboratory parameters, greater severity of illness, and a higher need for mechanical ventilation and vasoactive therapy. A retrospective study across China including 146,148 patients found AKI in $47.1 \%$ of sepsis cases [23]. Another study reported AKI is common even among patients without severe sepsis or shock $34 \%$ of non-severe community-acquired pneumonia developed AKI [28]. Literature reviews [4] reported that a 75\% increase in the intensive care unit (ICU)-treated severe sepsis from 1993 to 2001 in France.

In this study, the association of comorbidities, i.e. hypertension, diabetes, heart disease, the respiratory condition was not statistically significant. Although diabetes and number of comorbidities were found to be related to mortality in AKI patients in the previous studies [29] have shown that diabetes alone is an independent risk for acute kidney injury (AKI) and Diabetes significantly increases the overall morbidity and mortality, particularly by elevating the cardiovascular risk. e incidence of AKI was found to be higher in diabetic patients undergoing.

Nephrotoxic medication is the second cause of AKI in our study after sepsis 
accounting for $10 \%$ of our patients, these drugs include gentamycin, vancomycin and contrast dye. The most important adverse effects of aminoglycosides are nephrotoxicity, ototoxicity, and, rarely, neuromuscular blockade. Renal injury can occur in a substantial number of patients receiving an aminoglycoside, and whether it occurs depends on the patient's clinical condition and interactions with other nephrotoxic drugs. A study [30] reported nephrotoxic drugs to contribute to AKI in 19\% - $25 \%$ of cases in the ICU. And [31] reported AKI was observed in 609 patients $(61 \%)$ these numbers are different from our estimation probably these studies are focused on nephron toxic medication as the main study problem with a large number of patients and advanced diagnostic modalities.

In the current study, there was a significant relationship between initiation of renal replacement therapy and the outcome, we observed $45 \%$ mortality of patients received RRT comparing to $36 \%$ in patients managed conservatively.

We acknowledge that our study has several limitations. First, the relatively small number of patients studied in this research may not representative of other Centers in the same region, Secondly this study conducted in a single centre which may limit the statistical power where a small number of patients were included. Thirdly the decision to initiate renal replacement therapy was purely clinical and judgmental.

\section{Conclusions}

The high frequency and mortality of AKI in ICU patients cause a significant health challenge. Further studies should be required to adopt and formulate therapeutic strategies to prevent the occurrence of AKI in ICU patients and reduce mortality.

This study states that the development of AKI in ICU is an important risk factor for poor outcomes in the critical care setting. Prevention is the best method for avoiding AKI, with the early identification and recognition of high-risk patients.

\section{Acknowledgements}

I wish to express my sincere gratitude to our ICU staff for their support throughout the study, and also wish to express my sincere gratitude to the patients and their families who made this work possible.

\section{Declaration}

I declare that this work to be original and has not been published elsewhere, nor is it currently under consideration for publication elsewhere.

\section{Ethical Approve}

I declare this work has been approved by the authorized ethical committee in the ministry of health, the Republic of Sudan and by the scientific committee in 
Omdurman teaching hospital.

\section{Funding}

I declare this work to be self-funding by the authors, no financial support could have influenced the result of this work.

\section{Authors Contribution and Consents}

All the work in this research done by the authors listed. AS carried out the discussion and conclusion. NE carried out the data collecting and result. Both AS and NE contribute to the introduction, literature review, methodology and manuscript format. I confirm this manuscript has been revised and approved for submission by all the named authors.

\section{Availability of Data}

Data is available.

\section{Consent for Publication}

Not applicable.

\section{Conflicts of Interest}

I declare I have no conflicts of interest to disclose.

\section{References}

[1] Hoste, E.A.J., Clermont, G., Kersten, A., et al. (2006) RIFLE Criteria for Acute Kidney Injury Are Associated with Hospital Mortality in Critically Ill Patients: A Cohort Analysis. Critical Care, 10, Article No. R73.

[2] Kellum, J.A., Lameire, N., Aspelin, P., et al. (2012) Kidney Disease: Improving Global Outcomes (KDIGO) Acute Kidney Injury Work Group. KDIGO Clinical Practice Guideline for Acute Kidney Injury. Kidney International Supplements, 2, $1-138$.

[3] Himmelfarb, J. (2009) Acute Kidney Injury in the Elderly: Problems and Prospects. Semin. Nephrology, 29, 658-664. https://doi.org/10.1016/j.semnephrol.2009.07.008

[4] Brun-Buisson, C., Meshaka, P., Pinton, P., Vallet, B. and EPISEPSIS Study Group (2004) EPISEPSIS: A Reappraisal of the Epidemiology and Outcome of Severe Sepsis in French Intensive Care Units. Intensive Care Medicine, 30, 580-588.

[5] Yegenaga, I., Tuglular, S., Ari, E., et al. (2010) Evaluation of Sepsis/Systemic Inflammatory Response Syndrome, Acute Kidney Injury, and RIFLE Criteria in Two Tertiary Hospital Intensive Care Units in Turkey. Nephron Clinical Practice, 115, c276-c282. https://doi.org/10.1159/000313486

[6] Fox, C.S., Muntner, P., Chen, A.Y., Alexander, K.P., Roe, M.T. and Wiviott, S.D. (2012) Short-Term Outcomes of Acute Myocardial Infarction in Patients with Acute Kidney Injury: A Report from the National Cardiovascular Data Registry. Circulation, 125, 497-504. https://doi.org/10.1161/CIRCULATIONAHA.111.039909

[7] Fox, C.S., Muntner, P., Chen, A.Y., Alexander, K.P., et al. (2010) Use of Evi- 
dence-Based Therapies in Short-Term Outcomes of ST-Segment Elevation Myocardial Infarction and Non-ST-Segment Elevation Myocardial Infarction in Patients with Chronic Kidney Disease: A Report from the National Cardiovascular Data Acute Coronary Treatment and Intervention Outcomes Network registry. Circulation, 121, 357-365. https://doi.org/10.1161/CIRCULATIONAHA.109.865352

[8] Patschan, D. and Müller, G.A. (2016) Acute Kidney Injury in Diabetes Mellitus. International Journal of Nephrology, 2016, Article ID: 6232909. https://doi.org/10.1155/2016/6232909

[9] Mima, A. (2013) Diabetic Nephropathy: Protective Factors and a New Therapeutic paradigm. Journal of Diabetes and Its Complications, 27, 526-530. https://doi.org/10.1016/j.jdiacomp.2013.03.003

[10] Yu, S.M.W. and Bonventre, J.V. (2018) Acute Kidney Injury and Progression of Diabetic Kidney Disease. Advances in Chronic Kidney Disease, 25, 166-180. https://doi.org/10.1053/j.ackd.2017.12.005

[11] Saran, R., Robinson, B., Abbott, K.C., et al. (2017) US Renal Data System 2016 Annual Data Report: Epidemiology of Kidney Disease in the United States. American Journal of Kidney Diseases, 69, A7-A8.

[12] Waikar, S.S., Liu, K.D. and Chertow, G.M. (2008) Diagnosis, Epidemiology and Outcomes of Acute Kidney Injury. Clinical Journal of the American Society of Nephrology, 3, 844-861. https://doi.org/10.2215/CJN.05191107

[13] Mehta R.H., Grab, J.D., O’Brien, S.M., et al. (2006) Bedside Tool for Predicting the Risk of Postoperative Dialysis in Patients Undergoing Cardiac Surgery. Circulation, 114, 2208-2216. https://doi.org/10.1161/CIRCULATIONAHA.106.635573

[14] Erbay, A., Bodur, H., Akinci, E. and Colpan, A. (2005) Evaluation of Antibiotic Use in Intensive Care Units of A Tertiary Care Hospital in Turkey. Journal of Hospital Infection, 59, 53-61.

[15] Martínez-Salgado, C., López-Hernández, F.J. and López-Novoa, J.M. (2007) Glomerular Nephrotoxicity of Aminoglycosides. Toxicology and Applied Pharmacology, 223, 86-98. https://doi.org/10.1016/j.taap.2007.05.004

[16] Landau, D. and Kher, K.K. (1997) Gentamicin-Induced Bartter-Like Syndrome. Pediatric Nephrology, 11, 737-740. https://doi.org/10.1007/s004670050378

[17] Bertino, J.S., Booker, L.A., Franck, P.A., Jenkins, P.L., Franck, K.R. and Nafziger, A.N. (1993) Incidence of and Significant Risk Factors for Aminoglycoside-Associated Nephrotoxicity in Patients Dosed by Using Individualized Pharmacokinetic Monitoring. The Journal of Infectious Diseases, 167, 173-179. https://doi.org/10.1093/infdis/167.1.173

[18] Cartin-Ceba, R., Kashiouris, M., Plataki, M., Kor, D.J., Gajic, O., Casey, E.T., Cartin-Ceba, R., et al. (2012) Risk Factors for Development of Acute Kidney Injury in Critically Ill Patients: A Systematic Review and Meta-Analysis of Observational Studies. Critical Care Research and Practice, 2012, Article ID: 691013. https://doi.org/10.1155/2012/691013

[19] Kane-Gill, S.L., Sileanu, F.E., Murugan, R., Trietley, G.S., Handler S.M. and Kellum, J.A. (2015) Risk Factors for Acute Kidney Injury in Older Adults with Critical Illness: A Retrospective Cohort Study. American Journal of Kidney Diseases, 65, 860-869. https://doi.org/10.1053/j.ajkd.2014.10.018

[20] Nisula, S., Kaukonen, K.-M., Vaara, S.T., et al. (2013) Incidence, Risk Factors and 90-Day Mortality of Patients with Acute kidney Injury in finnish Intensive Care Units: The FINNAKI Study. Intensive Care Medicine, 39, 420-428. https://doi.org/10.1007/s00134-012-2796-5 
[21] Hoste, E.A.J., Kellum, J.A., Selby, N.M., et al. (2018) Global Epidemiology and Outcomes of Acute Kidney Injury. Nature Reviews Nephrology, 14, 607-625. https://doi.org/10.1038/s41581-018-0052-0

[22] Hashemian, S.M., et al. (2016) Outcome of Acute Kidney Injury in Critical Care Unit, Based on AKI Network. Tanaffos, 15, 89-95.

[23] Xu, X., Nie, S., Liu, Z.S., Chen, C.B., et al. (2015) Epidemiology and Clinical Correlates of AKI in Chinese Hospitalized Adults. Clinical Journal of the American Society of Nephrology, 10, 1510-1518. https://doi.org/10.2215/CJN.02140215

[24] van den Akker, J.P.C., Egal, M. and Groeneveld, J.A.B. (2013) Invasive Mechanical Ventilation as A Risk Factor for Acute Kidney Injury in the Critically Ill: A Systematic Review and Meta-Analysis. Critical Care, 17, Article No. R98.

https://doi.org/10.1186/cc12743

[25] Cortjens, B., Royakkers, A.A., Determann, R.M., et al. (2012) Lung-Protective Mechanical Ventilation Does Not Protect against Acute Kidney Injury in Patients without Lung Injury at Onset of Mechanical Ventilation. Journal of Critical Care, 27, 261-267. https://doi.org/10.1016/j.jcrc.2011.05.005

[26] Tanioku, T., Yoshida, A., Aratani, Y., Fujii, K. and Kawamata, T. (2020) Validation of Noninvasive Continuous Arterial Pressure Measurement by ClearSight SystemTM during Induction of Anesthesia for Cardiovascular Surgery. BMC Anesthesiology, 20, Article No. 176. https://doi.org/10.1186/s12871-020-01091-x

[27] Bagshaw, S.M., Uchino, S., Bellomo, R., et al. (2007) Septic Acute Kidney Injury in Critically Ill Patients: Clinical Characteristics and Outcomes. Clinical Journal of the American Society of Nephrology, 2, 431-439. https://doi.org/10.2215/CJN.03681106

[28] Murugan, R., Karajala-Subramanyam, V., Lee, M., et al. (2010) Acute Kidney Injury in Non-Severe Pneumonia Is Associated with An Increased Immune Response and Lower Survival. Kidney International, 77, 527-535. https://doi.org/10.1038/ki.2009.502

[29] Heung, M. and Koyner, J.L. (2015) Entanglement of Sepsis, Chronic Kidney Disease, and Other Comorbidities in Patients Who Develop Acute Kidney Injury. Seminars in Nephrology, 35, 23-37. https://doi.org/10.1016/j.semnephrol.2015.01.004

[30] Mehta, R.L., Pascual, M.T., Soroko, S., et al. (2004) Spectrum of Acute Renal Failure in the Intensive Care Unit: The PICARD Experience. Kidney International, 66, 1613-1621. https://doi.org/10.1111/j.1523-1755.2004.00927.x

[31] Ehrmann, S., Helms, J., Joret, A., et al. (2019) Nephrotoxic Drug Burden among 1001 Critically Ill Patients: Impact on Acute Kidney Injury. Annals of Intensive Care, 9, Article No. 106. https://doi.org/10.1186/s13613-019-0580-1

\section{Abbreviations}

AKI acute kidney injury

ICU intensive care unit 